\title{
Anatomical study of the anterior interosseous nerve
}

\author{
A. Jeon', M. Lee², D.W. Kim³, O.-Y. Kwon³, J.-H. Lee ${ }^{4}$ \\ 'Department of Anatomy/Catholic Institute for Applied Anatomy, College of Medicine, \\ The Catholic University of Korea, Seoul, Korea \\ ${ }^{2}$ School of Music and Arts, College of Music and Arts, Dankook University, Jukjeon, Korea \\ ${ }^{3}$ Department of Anatomy and Cell Biology, College of Medicine, Chungnam National University, Daejeon, Korea \\ ${ }^{4}$ Anatomy Laboratory, College of Sports Science, Korea National Sport University, Seoul, Korea
}

[Received: 8 January 2021; Accepted: 1 July 2021; Early publication date: 3 August 2021]

Background: The aim of this study is to investigate the location of nerves that innervate the flexor digitorum profundus (FDP), the flexor pollicis longus (FPL) and the pronator quadratus muscles. It also investigates the change in nerve location with hand movement.

Materials and methods: We studied 30 adult cadavers (17 males and 13 females) with a mean age of 69.5 years (range: 60-95 years). The reference line was from the humeral epicondylar line to the styloid process line of both the radius and ulnar bones. This study measured the anterior interosseous nerve (AIN) branch outpoint and the innervated muscle nerve entry point to the muscle belly. It also examines nerve position changes as related to making a fist.

Results: The reference line mean distance was $24.1 \pm 1.2 \mathrm{~cm}$. The median nerve branched into the AIN at $18.0 \pm 4.0 \%$. We found the most densely distributed section of the nerves' entry point to the muscle belly to be at a distance of $30 \%$ to $40 \%$ for the FDP and from 30\% to $40 \%$ for the FPL. Except for the FPL, the nerve branch outpoints and the FDP moved by $3.0 \%$, depending upon hand movements. Conclusions: The results of this study show that it will be necessary to consider the anatomy of the nerve location as it enters the muscle belly as well as how it changes with movement. (Folia Morphol 2022; 81, 3: 574-578)

Key words: anterior interosseous nerve, flexor digitorum profundus, flexor pollicis longus, pronator quadratus, nerve position change

\section{INTRODUCTION}

The anterior interosseous nerve (AIN) is a branch of the median nerve that supplies the deep muscle of the anterior forearm compartment. AIN syndrome, not common, was first described in 1952 [7]. AIN syndrome usually causes motor weakness of the thumb and index finger pincer movements and sometimes is accompanied by wrist pain. However, abnormalities resulting AIN syndrome sensation can vary, depending upon the situation $[10,14]$.

The treatment options for AIN syndrome can be either surgical or nonsurgical, but we usually consider the surgical option if nonsurgical options have not previously been done within 4 to 6 months [8]. The anatomical aspect, even if there is an abnormality in the muscles and pain innervated by AIN, the observation range should be kept in mind for a wide 
region from the brachial plexus to the forearm. This above reason will seriously think about the possible entrapment spot, and will apply specifically for the conservative nonsurgical treatment. When treating with conservative treatment, the faster the treatment period will be more effective. An earlier report shows that manual reduction of an AIN injury is effective in bringing about recovery [9]. However, a more useful therapeutic approach is possible after understanding how muscle movements can affect the anatomical location of both the nerves and muscles.

Nerve transfer has become a vital method in the reconstruction of hand function, especially in the reconstruction of the intrinsic muscles of the hand following injury to the ulnar or median nerve. The AIN is a common donor site for the ulnar nerve or the branch of the median nerve, resulting in a good clinical outcome [4-6].

The aim of this study is to investigate the location of nerves that innervate the flexor digitorum profundus (FDF), the flexor pollicis longus (FPL) and the pronator quadratus muscles. It also investigates how movement changes nerve location.

\section{MATERIALS AND METHODS}

We studied 30 unembalmed adult cadavers (17 males and 13 females) with a mean age of 69.5 years (range: 60-95 years). The specimens chosen had no history of forearm fracture or arm surgery.

For the measurements, on the anterior view, we identified the most prominent point of the medial epicondyle of the humerus (MEH), and of the lateral epicondyle of the humerus (LEH) before doing the dissection. On the distal view, we also identified the most distal point of the styloid process of the radius (SPR) and of the ulna (SPU). A line connecting the midpoints of the MEH and the LEH, as well as the midpoint of the SPR and the SPU, was used as a starting point reference line from proximal to distal (Fig. 1).

All experiments were conducted in the anatomy laboratory of the medical school. With the body in a supine position, we first dissected the skin and fat tissue to expose the pronator teres muscle. We attempted to locate the median nerve and carefully traced it distally to find the AIN. After finding the AIN, we carefully dissected it to examine the nerve branches around it in detail. In addition, in order to examine the AIN, the insertion site of pronator teres was cut and observed. We investigated the

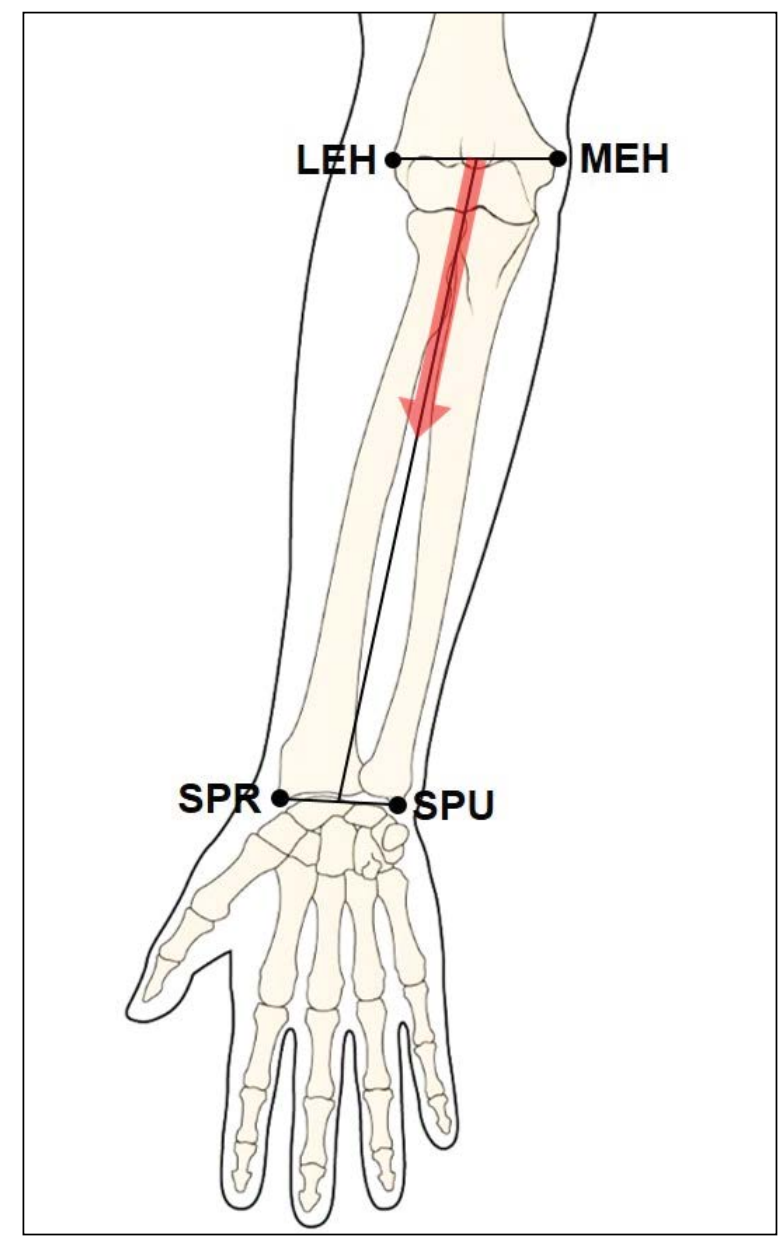

Figure 1. Illustration showing the reference line; LEH — lateral epicondyle of humerus; MEH - medial epicondyle of humerus; SPR - styloid process of radius; SPU — styloid process of ulna; red arrow - measuring direction.

target muscle type, the AIN nerve branch number that innervated each muscle, and the morphological location of nerves entering the muscle belly (Fig. 2). The measuring variables are as follow:

- the reference line length;

- the location of the point where the AIN divides from the median nerve;

- the location of the branch nerve outpoints that innervate the FPL and the FDF;

- the crossing point of the AIN with the superior border of pronator quadratus.

Additionally, to examine how movement changes nerve position, we performed the following steps on three unembalmed cadavers (Fig. 3).

1. After dissection, we measured the nerve entry point to the FPL and FDP when the palm was open;

2. After dissection, we measured the median branch outpoint to the AIN; 


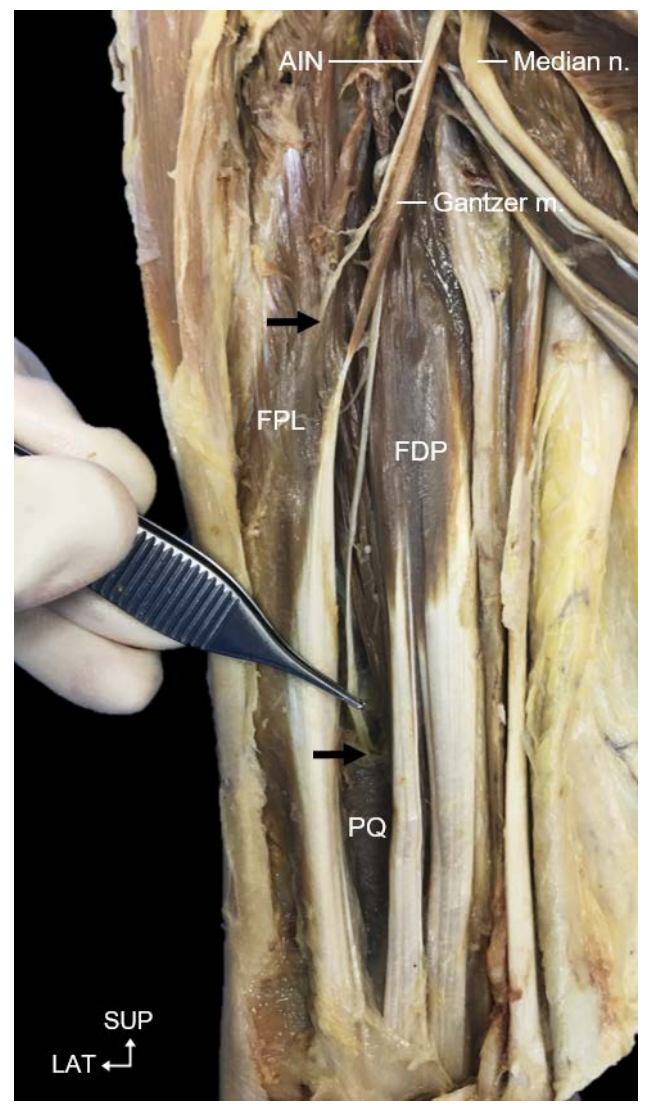

Figure 2. Photographs of the dissected forearm of fixed specimen; $\mathrm{PO}$ - pronator quadrates; FPL — flexor pollicis longus; FDP flexor digitorum profundus; AIN — anterior interosseous nerve; SUP — superior; LAT — lateral; arrow — nerve entering point to the muscle belly.

3. The measured points were marked with a thumbtack;

4. With the closed hand fist position, we measured the median branch outpoint to the AIN;

5. After opening the fist, we measured the degree of change in the nerve position.

A single observer took all measurements with a measuring tape and digital callipers (resolution $0.01 \mathrm{~mm}, \mathrm{CD}-20 \mathrm{PSX}$, Mitutoyo, Japan). Data was analysed using SPSS software version 23.0 (IBM SPSS Inc., Chicago, IL, USA). Comparisons between male and female cadavers were performed using the t-test. $P$ values of less than 0.05 were considered statistically significant. The present study was conducted in accordance with the Declaration of Helsinki

\section{RESULTS}

The mean distance of the reference line was $24.1 \pm 1.2 \mathrm{~cm}$. The median branched into the AIN at $18 \pm 4.0 \%$. No significant distant differences were

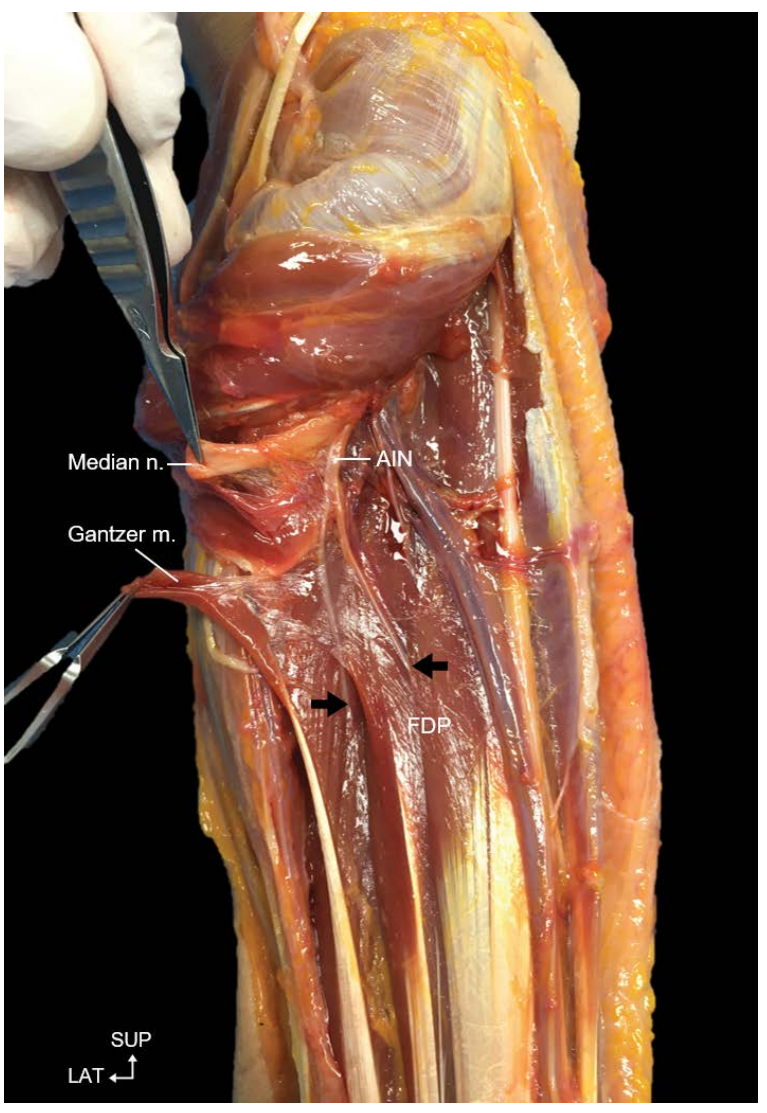

Figure 3. Photographs of the dissected forearm of unembalmed specimen; FDP — flexor digitorum profundus; SUP — superior; LAT — lateral; arrow — nerve entering point to the muscle belly.

found in the reference line between males and females or between the right and left forearm $(p \geq 0.05)$.

We investigated the nerve entry points to the muscle belly in this study. For the FPL, the number of nerve entry points ranged from 2 to 5 , and the distribution ratio was $31.0 \%, 37.9 \%, 24.1 \%$ and $7.0 \%$. This study had 10 sections, based on reference length, and it examined which sections were densely distributed. After checking the raw data, we found the most densely distributed section was distance from $40 \%$ to $50 \%$ and distance from $30 \%$ to $60 \%$ sections was distributed about $94.8 \%$ (Table 1).

For the FDP, the number of nerve points ranged from 2 to 5 , and the distribution ratio was $25.0 \%$, $46.4 \%, 21.4 \%$ and $7.2 \%$. Additionally, this study was divided into 10 sections, based on the reference length, and it examined which sections were densely distributed. After checking the raw data, we found the most densely distributed sections had a distance of $30 \%$ to $40 \%$ (Table 2 ).

The branch outpoint from the median nerve in the open palm position was $26.8 \pm 1.9 \%$. Howev- 
Table 1. The mean value of the distributed frequency of nerve entry points to the flexor pollicis longus muscle belly

\begin{tabular}{lcc}
\hline Structures & $\begin{array}{c}\text { Mean } \pm \text { SD } \\
{[\mathbf{c m}]}\end{array}$ & $\begin{array}{c}\text { Distributed frequency } \\
\text { (\%) }\end{array}$ \\
\hline Section 3 (20-30\%) & $26.0 \pm 0.2$ & 1.4 \\
Section 4 (30-40\%) & $36.2 \pm 2.2$ & 25.2 \\
Section 5 (40-50\%) & $45.2 \pm 3.5$ & 58.8 \\
Section 6 (50-60\%) & $53.1 \pm 2.7$ & 10.8 \\
Section 7 (60-70\%) & $64.0 \pm 0.1$ & 2.6 \\
Section 8 (70-80\%) & $71.5 \pm 0.1$ & 1.2 \\
\hline
\end{tabular}

SD - standard deviation
Table 2. The mean value of the distribution frequency of nerve entry points to the flexor digitorum profundus muscle belly

\begin{tabular}{lcc}
\hline Structures & $\begin{array}{c}\text { Mean } \pm \text { SD } \\
\text { [cm] }\end{array}$ & $\begin{array}{c}\text { Distributed frequency } \\
(\%)\end{array}$ \\
\hline Section 3 (20-30\%) & $27.2 \pm 1.6$ & 9.3 \\
Section 4 (30-40\%) & $35.1 \pm 2.7$ & 60.1 \\
Section 5 (40-50\%) & $43.0 \pm 2.3$ & 11.9 \\
Section 6 (50-60\%) & $54.9 \pm 3.3$ & 11.7 \\
Section 7 (60-70\%) & $62.3 \pm 2.1$ & 7.0 \\
Section 8 (70-80\%) & - & - \\
\hline
\end{tabular}

SD - standard deviation

Table 3. Nerve position change in accordance with muscle contraction (unit: \%)

\begin{tabular}{cccccccc}
\hline & \multicolumn{7}{c}{ Variable } \\
\cline { 2 - 7 } & A & B & C & D & E & F \\
\hline Value & $26.8 \pm 1.9$ & $29.9 \pm 3.1$ & $39.1 \pm 2.7$ & $44.3 \pm 2.7$ & $45.1 \pm 3.0$ & $45.4 \pm 4.3$ \\
\hline
\end{tabular}

A - branch outpoint from median nerve in an open hand position; B - branch outpoint from median nerve in a closed fist hand position; $C$ - nerve entry point of the flexor digitorum profundus muscle belly in an open hand position; $\mathrm{D}$ - nerve entry point of the flexor digitorum profundus muscle belly in a closed fist position; $\mathrm{E}$ - nerve entry point of the flexor pollicis longus muscle belly in a palm opened position; $\mathrm{F}$ - nerve entry point in the flexor pollicis longus muscle belly in a fist closed position

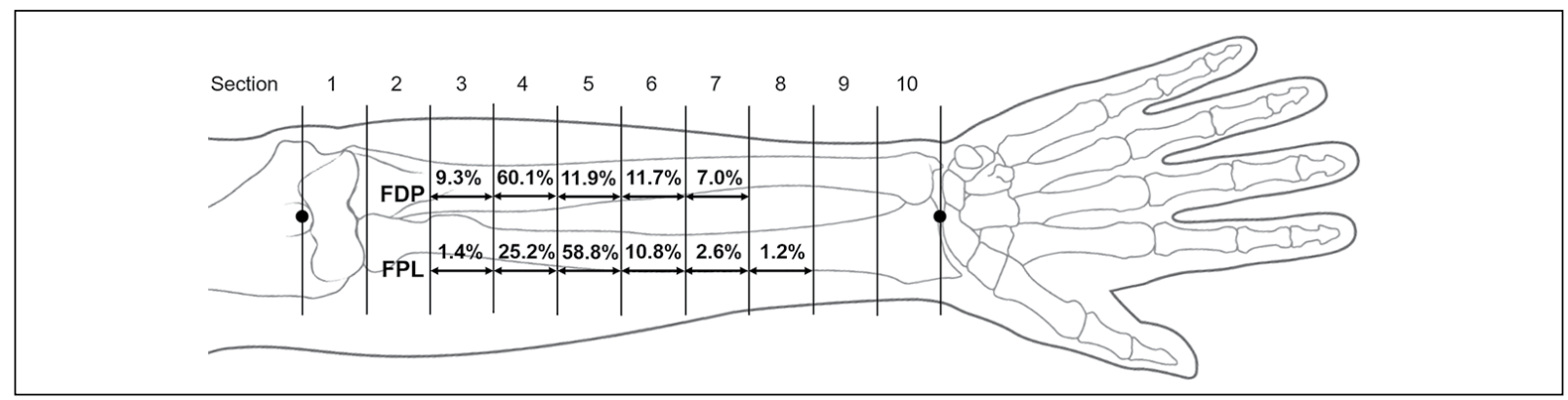

Figure 4. Degree of nerve distribution to enter the muscle belly on the reference line; FDP — flexor digitorum profundus; FPL — flexor pollicis longus.

er, after making a fist, the nerve change point was $29.9 \pm 3.1 \%$. So, except for the FPL, the nerve outpoint branch and FDP were moved 3.0\% in accordance with hand movement (Table 3 ). The crossing point of the AIN with superior border of pronator quadratus was located at $76.1 \pm 4.9 \%$ from the reference line. And the Gantzer muscle was found in 16 out of 60 specimens (Figs. 2, 3).

\section{DISCUSSION}

The AIN branched out under the pronator teres from the median nerve. This location might have caused AIN entrapment from the position where the nerves are divided. It is thought that the condition of the surrounding muscles, like a pronator teres, will cause nerve pressure. In this study, the median nerve runs to the anterior aspect of the hand and then branches out to the AIN at $4.4 \pm 1.1 \mathrm{~cm}$ from the reference line. Previous studies have reported that the AIN branches out between 2.3 and $8.0 \mathrm{~cm}$ in Sunderland [11], $5.4 \mathrm{~cm}$ in Tubbs et al. [13], $8.0 \mathrm{~cm}$ in Benzel [1], $4.5 \mathrm{~cm}$ in Vincelet et al. [15] and $5.2 \mathrm{~cm}$ in Caetano et al. [3]. A number of cases reported about $5.0 \mathrm{~cm}$ regardless of population. The branch outpoint will become reference material for the treatment of AIN syndrome.

The integrated trigger point hypothesis postulates that motor endplates release excessive acetylcholine in myofascial pain, which is evidenced histopathologically by the presence of sarcomere shortening [2]. Target muscle and nerve production of abnormal acetylcholine is appropriately stimulated. At this time, the effect can be expected by treatment the nerve entered point of muscle belly. The recommended site 
of recovery is $40.0-50.0 \%$ for the FPL and $30.0-40.0 \%$ for the FDP in this study (Fig. 4). The nerve entry point to the muscle belly is reported in this study. The information on the muscle belly nerve entry point can be used as helpful data for muscle and nerve relaxation during the clinical approach.

Clinically, the Gantzer muscle can also cause AIN syndrome [12]. Other anatomical studies have also indicated a relation between the Gantzer muscle and AIN syndrome [3]. The Gantzer muscle was found in 16 out of 60 specimens from all 8 cadavers and on both sides. In all cases found, this was independently innervated, and the AIN passed under the Gantzer muscle. This locational cause can affect the AIN syndrome according Gantzer muscle existence.

In 3 out of 60 specimens, the flexor digitorum superficialis was innervated by a branch of the AIN. Although it was difficult to find previous studies, this will provide reference material for muscle analysis related to AIN syndrome. One of the limitations of this study is our inability to examine more specimens. The fact that there was a $5.0 \%$ variation in FDP muscle nerve position related to whether the hand is open or closed into a fist is considered to be meaningful for the anatomical study of nerve position changes as related to movement.

\section{CONCLUSIONS}

The results of this study found that nerve location entered muscle belly and also nerve location related to movement with anatomical various investigation will be considered to treat in clinical practice.

\section{Acknowledgements}

The authors would like to thank the cadaver donors and their families who participated in the donation programme. This work was supported by the National Research Foundation of Korea (NRF) grant funded by the Korea government (MSIT) (No. 2019R1C1C1008845).

\section{Conflict of interest: None declared}

\section{REFERENCES}

1. Benzel EC. Practical approaches to peripheral nerve surgery. American Association of Neurological Surgeons. 1992: 51-63.

2. Bron C, Dommerholt JD. Etiology of myofascial trigger points. Curr Pain Headache Rep. 2012; 16(5): 439-444, doi: 10.1007/ s11916-012-0289-4, indexed in Pubmed: 22836591.
3. Caetano EB, Vieira LA, Sabongi Neto JJ, et al. Anterior interosseous nerve: anatomical study and clinical implications. Rev Bras Ortop. 2018; 53(5): 575-581, doi: 10.1016/j. rboe.2018.07.010, indexed in Pubmed: 30245997.

4. Dunn JC, Gonzalez GA, Fernandez I, et al. Supercharge end-to-side nerve transfer: systematic review. Hand (NY). 2021; 16(2): 151-156, doi: 10.1177/1558944719836213, indexed in Pubmed: 30924361.

5. Frank K, Englbrecht M, Koban KC, et al. Nerve transfer of the anterior interosseous nerve to the thenar branch of the median nerve - an anatomical and histological analysis. J Plast Reconstr Aesthet Surg. 2019; 72(5): 751-758, doi: 10.1016/j.bjps.2018.12.017, indexed in Pubmed: 30600157.

6. Head LK, Zhang ZZ, Hicks K, et al. Evaluation of intrinsic hand musculature reinnervation following supercharge end-to-side anterior interosseous-to-ulnar motor nerve transfer. Plast Reconstr Surg. 2020; 146(1): 128-132, doi: 10.1097/PRS.0000000000006903, indexed in Pubmed: 32590654.

7. Kiloh LG, Nevin S. Isolated neuritis of the anterior interosseous nerve. Br Med J. 1952; 1(4763): 850-851, doi: 10.1136/bmj.1.4763.850, indexed in Pubmed: 14916168

8. Kodama N, Ando K, Takemura Y, et al. Treatment of spontaneous anterior interosseous nerve palsy. J Neurosurg. 2020; 132(4): 1243-1248, doi: 10.3171/2018.11. JNS181609, indexed in Pubmed: 32539243.

9. Li HM, Liu XJ. [Manual reduction of children's Monteggia fractures associated with anterior interosseous nerve injury]. Zhongguo Gu Shang. China J Orthopaedics Traumatology. 2014; 27(10): 862-865, indexed in Pubmed: 25739256.

10. Nakano KK, Lundergran C, Okihiro MM. Anterior interosseous nerve syndromes. Diagnostic methods and alternative treatments. Arch Neurol. 1977; 34(8): 477-480, doi: 10.1001/archneur.1977.00500200037007, indexed in Pubmed: 196582.

11. Sunderland $S$. The intraneural topography of the radial, median and ulnar nerves. Brain. 1945; 68: 243-299, doi: 10.1093/brain/68.4.243, indexed in Pubmed: 20982793.

12. Tabib W, Aboufarah F, Asselineau A. Compression of the anterior interosseous nerve by Gantzer's muscle. Chirurgie de la Main. 2001; 20(3): 241-246, doi: 10.1016/s12973203(01)00041-5, indexed in Pubmed: 11496612.

13. Tubbs RS, Custis JW, Salter EG, et al. Quantitation of and superficial surgical landmarks for the anterior interosseous nerve. J Neurosurg. 2006; 104(5): 787-791, doi: 10.3171/ jns.2006.104.5.787, indexed in Pubmed: 16703884.

14. Ulrich D, Piatkowski A, Pallua N. Anterior interosseous nerve syndrome: retrospective analysis of 14 patients. Arch Orthop Trauma Surg. 2011; 131(11): 1561-1565, doi: 10.1007/s00402-011-1322-5, indexed in Pubmed: 21611763.

15. Vincelet $Y$, Journeau $P$, Popkov $D$, et al. The anatomical basis for anterior interosseous nerve palsy secondary to supracondylar humerus fractures in children. Orthop Traumatol Surg Res. 2013; 99(5): 543-547, doi: 10.1016/j.otsr.2013.04.002, indexed in Pubmed: 23916783. 